\title{
Frequency of Panel Reactive Antibodies (PRA) among Renal Transplant Recipients and its Effect Modifiers
}

\author{
Muhammad Hussain, Muhammad Mukarram Bashir, Hamid Nawaz Tipu, Muhammad Dawood and Mustajab \\ Alam \\ Department of Immunology, Armed Forces Institute of Pathology, Rawalpindi, Pakistan
}

\begin{abstract}
Objective: To determine frequency of panel reactive antibodies among renal transplant recipients and its effect modifiers. Study Design: A cross-sectional study. Place and duration of study: Department of Immunology, Armed Forces Institute of Pathology from October 2016 to October 2017. Methodology: One hundred and sixty-two (162) patients, who were referred to Department of Immunology for pre-transplant workup for kidney transplantation of both genders and Pakistani nationality. Informed consents were taken and detailed history were recorded. Frequency and percentages were calculated for panel reactive antibodies, blood transfusion, pregnancy and previous transplant were noted and Chi-square test was applied.

Results: One hundred and sixty-two (162) patients including 141 males and 21 females were analysed and 48 patients (30\%) were positive for panel reactive antibodies (PRA). Of 141 male patients analyzed, 35 were positive for PRA, which were about $25 \%$. Twenty-one females were tested for PRA and 13 female patients were positive that is about $62 \%$ of the analysed population. Out of the total 141 males, $20(14 \%)$ had blood transfusion and of these $11(55 \%)$ were positive for PRA. Without history of transfusion, only $9(7 \%)$ were positive for PRA. Out of 21 females, 10 were positive for blood transfusion, out of which $6(60 \%)$ were positive for PRA. Without history of blood transfusion, 7 (64\%) were positive for PRA. Out of 21 females, 20 had history of pregnancy. Out of whom, $13(65 \%)$ were positive for PRA. Two patients (one male and one female) were with history of previous transplant and both were positive for PRA.

Conclusion: A significant number of patients were sensitised with panel reactive antibodies waiting for renal transplant. The PRA was more common in recipients who were prone to effect modifiers such as pregnancy, blood transfusion and re-transplant. These risk factors were mostly present in combination, which also suggests their synergistic effects on PRA synthesis.
\end{abstract}

Key Words: Blood transfusion, Effect modifiers, Panel reactive antibodies, Pregnancy, Kidney transplant, Re-transplant.

How to cite this article: Hussain M, Bashir MM, Tipu HN, Dawood M, Alam M. Frequency of Panel Reactive Antibodies (PRA) among Renal Transplant Recipients and its Effect Modifiers. J Coll Physicians Surg Pak 2020; 30(09):966-969.

\section{INTRODUCTION}

Panel reactive antibodies (PRA) are defined as the preexisting antibodies against human leukocyte antigens (HLA) in the serum of potential allograft recipients. ${ }^{1}$ Presence of antibodies against HLA molecules, which may be directed against HLA class I and class II antigens, is a risk factor for hyper acute rejection and graft loss. ${ }^{2}$

MHC class I related chain (MIC) antigens are surface glycoprotein and are expressed on endothelial cells, epithelial cells, dendritic cells, fibroblasts and on many tumor cells but not on lymphocytes. ${ }^{3}$ Antibodies against these MIC antigens are associated with increased incidence of graft loss in the recipients who are fully HLA matched. ${ }^{4}$

Correspondence to: Dr. Muhammad Hussain, Department of Immunology, Armed Forces Institute of Pathology, Rawalpindi, Pakistan

E-mail: capthussainkk@yahoo.com

Received: May 21, 2019; Revised: January 15, 2020;

Accepted: January 30, 2020

DOI: https://doi.org/10.29271/jcpsp.2020.09.966
Anti HLA antibodies may develop with previous blood transfusion, pregnancy and transplant; and degree of sensitisation is stronger and more prolonged when different factors act together. ${ }^{5} \mathrm{~A}$ high PRA means that the individual is primed to react immunologically against a large proportion of the population. ${ }^{2}$ Sensitisation has a major impact on patient mortality and morbidity due to prolonged waiting time and may delay transplantation. ${ }^{6}$ Each population has different demography of HLA antigens and so the PRA test will be different in different ethnic groups. $^{7}$

Presence of panel reactive antibodies in pre-transplant recipients is associated with increased incidence of early/latent graft loss. ${ }^{8}$ A study conducted by Meng et al., evaluated the presence of anti-HLA antibodies in kidney transplant recipients in 73 PRA pre-sensitised recipientscompared with 81 non-sensitisedrecipients; and clinical follow-up was recorded at 24 months. In the 73 sensitised individuals, the rate of rejection and graft survival was $35.6 \%$ and $80.8 \%$, respectively as compared to 81 non-sensitised cases, where rejection and graft survival was $18.5 \%$ and $95.1 \%$, respectively, showing a significant difference. ${ }^{9}$ 
The subject has been an area of extensive research with clinical application of paramount importance. In Pakistan, no previous study, to the best of our knowledge, has so far been conducted to find out and document the panel reactive antibodies and its effect modifiers such as blood transfusion, pregnancy, re-transplant and infections.

The aim of this study was to determine the individual and collectivesignificance of PRA and its effectmodifiers in renal pre-transplant compatibility of donors with potential recipients.

\section{METHODOLOGY}

A descriptive cross-sectional study was conducted from October 2016 to October 2017 in which 162 patients, who were referred to the Department of Immunology, AFIP for pre-transplant workup of kidney transplantation, were included. Patients of Pakistani nationality waiting for renal transplant were included and those with nationality other than Pakistan were excluded. Informed consent was taken and detailed history was recorded. In this test, $3 \mathrm{ml}$ of patient's serum was mixed with the beads coated with MHC molecules. If patients' serum contains anti-HLA antibodies, it will bind to these beads and secondary fluorescence labelled antibody attached to these anti-HLA antibodies. These fluorescence labelled antibodies are detected by Luminex machine.

Frequency and percentages were calculated for gender and PRA, Chi-square test was applied and effect modifiers such as transfusion, pregnancy and previous transplant were noted as these are the strong effect modifiers for HLA antibodies.

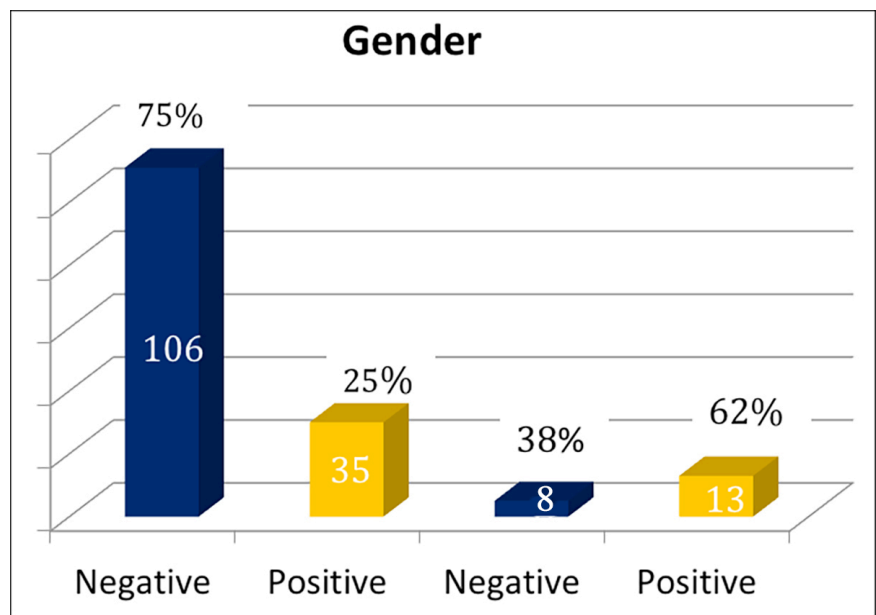

Figure 1: 141 male patients were analysed and among them 35 (25\%) were positive for panel reactive antibodies. Twenty-one females were tested for PRA and 13 (62\%) female patients were positive (Male $n=141$, female $n=21$ ).

\section{RESULTS}

One hundred and sixty-two patients, including 141 males and 21 females, were studied. The panel reactive antibodies were present in $48(30 \%)$ recipients, comprising of 17 (35\%) class I, $10(21 \%)$ class II, 16 (33\%) both class I and class II and 5 (10\%) MIC antibodies. Among 141 male patients, 35 (25\%) were positive for panel reactive antibodies. Twelve male patients were positive for class I, 9 for class II, 9 for both class I and class II and 5 for MIC. Twenty-one females were tested for PRA and 13 $(62 \%)$ female patients were positive (Figure 1).

Out of these 13 positive females, 5 were positive for class I, 1 for class II and 7 were positive for both class I and class II, respectively. No female was positive for MIC antibodies.

Out of total 141 males, 20 (14\%) had received blood transfusion in the past; and among these 20 transfusion positive, 11 $(55 \%)$ were positive for PRA. Significantly, male patients with transfusion ( $p=$ value 0.001 ). Out of total 21 females, 10 were positive for blood transfusion; and out of these, 6 (60\%) were positive for PRA and without history of blood transfusion, 7 (64\%) were positive for PRA. Female patients with transfusion history were not in statistically significant proportion ( $p=$ value of 0.86 ). Out of 21 females, 20 were positive for pregnancy. Out of these 20 females, 13 (62\%) were positive for PRA $(p=0.19)$. Two patients, one male and one female, were with history of previous transplant and both were positive for panel reactive antibodies.

High number of positive results in females was because they are more prone to effect modifiers such as pregnancy and blood transfusion. A repeat transplant is also a strong risk factor for development of panel reactive antibodies. Males having positive for panel reactive antibodies had less history regarding effect modifiers, suggesting some other factors for de novo synthesis of panel reactive antibodies such as infections.

\section{DISCUSSION}

Data regarding frequency of panel reactive antibodies among renal transplant recipients is lacking in our region, especially in Pakistan. Presence of antibodies against HLA and non-HLA molecules, which may be directed against HLA class I and class II antigens and MIC antigens is a risk factor for hyper-acute, acute, chronic rejection and graft loss. ${ }^{1}$ Before transplant, panel reactive antibody (PRA) is done to identify sensitized patients. ${ }^{1}$

A study conducted by Mishra et al, in which 52 patients (male-36, femele-16) were analysed at Transplant Centre in Pune, India. The age of patients ranged from 11 to 53 years (mean 34.7 years). ${ }^{1}$ The study of 52 subjects revealed $23 \%$ positivity for panel reactive antibodies ${ }^{1}$. In the sensitised category, PRA positivity was higher for class I than for class II $^{1}$. This study has limitations of small sample size. This study showed 30\% positivity for PRA, which is a little higher. Furthermore, PRA against class I is more positive than those against class II, which is same as in Indian study conducted by Mishra etal. ${ }^{1}$

In Romanian study, pre-transplant evaluation of 500 subjects were carried out which illustrated that out of these, 145 patients are sensitised. Among these sensitised individuals, 85 patients (17\%) presented anti-HLA class I antibodies, 19 patients (4\%) presented anti HLA class II antibodies and in 41 (8\%) subjects, both class I and class II antibodies were detected. ${ }^{5}$ In the present study, $30 \%$ positivity was noted which is nearly equal to Roma- 
nian population. PRA distribution is also similar to that in this study. However, contrary to the present study, it lacked discussion of effectmodifiers.

A study conducted at Baskent University, Istanbul Practice and Research Hospital, from January 2011 till December 2012, 620 patients diagnosed with end-stage renal disease and waiting for a renal transplant were analysed in this retrospective study at Transplantation Department. ${ }^{10}$ It was found that panel reactive antibodies screening positivity in $20.4 \%$ of patients on the renal transplant waiting list. ${ }^{6}$ Panel reactive antibodies were identified more against anti-HLA class II antibodies in contrast to our study, which has more anti-HLA antibodies against class I probably due to genetic variation in different population.

Another study included 2,517 patients ( 1,428 males and 1,089 females), age ranging from 2 to 82 years, with chronic kidney failure awaiting renal transplant. They were tested for PRA by Tissue Typing Laboratory in Tepecik Teaching and Research Hospital (TRH), Turkey between January 2008 and June $2014 .{ }^{11} \mathrm{~A}$ total of 1,020 patients (40.52\%) were PRA positive on the kidney transplant waiting list. Anti-HLA antibodies for class I were positive in $316(13 \%)$ patients, class II in 221 (09\%) and both class I and class II positive in 483 (19\%) patients; while 1,497 (59.48\%) of the patients were PRA negative. ${ }^{11}$ The present study has less PRA positivity as compared to the study, but PRA class I and class II distribution pattern was same. Less PRA positivity in the presentstudy may be because of small samplesize.

A study conducted by Zou et al. at University of Texas on 1,910 patients revealed that MIC antibodies were present in 217 (11\%) individuals. ${ }^{12}$ This study showed similar result as $9 \%$ of patients on transplant waiting list are positive for anti-MIC antibodies.

A study conducted by Darrel et al. analysed 1,816 non-pregnant females and 3,992 pregnant females. Among female donors, who reported a previous pregnancy, 973 out of 3,992 (24.4\%) were positive for anti-HLA antibodies. He also observed that with each pregnancy, probability of sensitisation increases. ${ }^{13}$ In this study, $62 \%$ of females with history of previous pregnancies were positive for panel reactive antibodies, which is comparatively higher compared to the study conducted by Darrel et al. This may be because of poor antenatal care and frequent blood transfusions.

A study conducted by Hyun in Europe determined PRA identification on 674 patients ( 354 males and 320 females). In his study, he showed female to male ratio $60.3 \%$ vs. $34.2 \%{ }^{14}$ whereas, this study showed female to male ratio $65 \%$ vs. $25 \%$. He also observed that PRA positive rates were significantly higher in patients with transfusion (33\%), pregnancy $(71 \%)$, or previous transplantation $(77 \%)$ than in controls without any identifiable sensitization events $(5.6 \%) .{ }^{14}$ This study showed that PRA positivity in patients with history of transfusion is $57 \%$, with previous pregnancy $60 \%$ and two patients had previous transplant and both were positive for PRA. This shows our population is prone to more panel reactive antibodies when compared to European population.

\section{CONCLUSION}

Panel reactive antibodies are the essential test for the workup of renal transplantation and also used for monitoring of post-transplant sensitisation. A significant number of patients were sensitised with panel reactive antibodies, waiting for renal transplant. The panel reactive antibodies were more common in recipients, who were more prone to effect modifiers such as pregnancy, blood transfusion and re-transplant as compared to those not prone to them. These risk factors are mostly present in combination; also suggesting the synergistic effects on the synthesis of panel reactive antibodies.

\section{CONFLICT OF INTEREST:}

This study has no conflict of interest to be declared by any author.

\section{ETHICALAPPROVAL:}

This study is approved by Ethical Review Committee, Armed Forces Institute of Pathology (AFIP) Rawalpindi and ethical approval was obtained prior to initiation of the research work.

\section{PATIENTS' CONSENT:}

Informed consents were taken from all patients about the study and detailed history was recorded.

\section{AUTHORS' CONTRIBUTION:}

$\mathrm{MH}$ : Concept and write-up.

MMB: Design.

HNT, MD: Critical review.

$A B$ : Data collection.

MA: Interpretation of data.

\section{REFERENCES}

1. Mishra MN, Baliga KV. Significance of panel reactive antibodies in patients requiring kidney transplantation. Saudi J Kidney Dis Transpl 2013; 24(3):495-9.

2. Lim WH, Chapman JR, Wong G. Peak panel reactive antibody, cancer, graft, and patient outcomes in kidney transplant recipients. Transplantation 2015; 99(5): 1043-50.

3. Oremo JAA, Zhang Xiao, Zhu Sha. MHC class I related chain $A \& B$ ligands are differently expressed in cancer cell lines. Immunol Disordimmunother 2016; 1(1):103.

4. Risti M, Bicalho MD. MiCA and NKG2D: There is an impact on kidney transplant outcome. Frontiers in immunology 2017; 8:179.

5. Moise AM, Nedelcu D, Toader A, Sora M, Tica A, Ferastraoaru DE, et al. Cytotoxic antibodies-valuable prognostic factor for long term kidney allograft survival. J Med Life 2010; 3(4):390-5.

6. Keven K, Sengul S, Celebi ZK, Tuzuner A, Yalcin F, Duman $\mathrm{T}$, et al. Kidney transplantation in immunologically high-risk patients. Transplant Proc 2013; 45(3):919-22.

7. Picascia A, Infante T, Napoli C. Luminex and antibody detection in kidney transplantation. Clin ExpNephrol 2012; 16(3):373-81.

8. Lachmann N, Todorova K, Schulze H, Schönemann C. Luminex and its applications for solid organ transplant- 
ation, hematopoietic stem cell transplantation, and transfusion. Transfusion Medicine and Hemotherapy 2013; 40(3):182-9.

9. Meng HL, Jin XB, Li XT, Wang HW, Lü J. Impact of human leukocyte antigen matching and recipients' panel reactive antibodies on two-year outcome in presensitized renal allograft recipients. Chin Med J (Engl) 2009; 122(4):420-6.

10. İnal A, Özçelik Ü, Ogan EU, Külah E, Demirağ A. Analysis of panel reactive antibodies in renal transplant recipients detected by luminex: A single-center experience. Exp Clin Transplant 2016; 14(4):401-4.

11. Böbrek PR, Fonksiyonu YO. The effect of panel reactive antibody results on graft functions of patients with chronic kidney failure 2015.

12. Zou Y, Stastny P, Süsal C, Döhler B, Opelz G. Antibodies against MICA antigens and kidney-transplant rejection. $N$ Engl J Med 2007; 357(13):1293-300.

13. Triulzi DJ, Kleinman S, Kakaiya RM, Busch MP, Norris PJ, Steele WR, et al. The effect of previous pregnancy and transfusion on HLA alloimmunization in blood donors: Implications for a transfusion-related acute lung injury risk reduction strategy. Transfusion 2009; 49(9):1825-35.

14. Hyun J, Park KD, Yoo Y, Lee B, Han BY, Song EY, et al. Effects of different sensitization events on HLA alloimmunization in solid organ transplantation patients. Transplant Proc 2012; 44(1):222-5. 